\title{
On the Domination Polynomial of Some Graph Operations
}

\author{
Saeid Alikhani ${ }^{1,2}$ \\ ${ }^{1}$ Department of Mathematics, Yazd University, Yazd 89195-741, Iran \\ ${ }^{2}$ School of Mathematics, Institute for Research in Fundamental Sciences (IPM), P.O. Box 19395-5746, Tehran, Iran \\ Correspondence should be addressed to Saeid Alikhani; alikhani206@gmail.com
}

Received 9 June 2013; Accepted 28 July 2013

Academic Editors: L. Feng and J. Siemons

Copyright (C) 2013 Saeid Alikhani. This is an open access article distributed under the Creative Commons Attribution License, which permits unrestricted use, distribution, and reproduction in any medium, provided the original work is properly cited.

Let $G$ be a simple graph of order $n$. The domination polynomial of $G$ is the polynomial $D(G, \lambda)=\sum_{i=0}^{n} d(G, i) \lambda^{i}$, where $d(G, i)$ is the number of dominating sets of $G$ of size $i$. Every root of $D(G, \lambda)$ is called the domination root of $G$. In this paper, we study the domination polynomial of some graph operations.

\section{Introduction}

Let $G$ be a simple graph. For any vertex $v \in V$, the open neighborhood of $v$ is the set $N(v)=\{u \in V \mid u v \in E\}$ and the closed neighborhood is the set $N[v]=N(v) \cup\{v\}$. For a set $S \subseteq V$, the open neighborhood of $S$ is $N(S)=\bigcup_{v \in S} N(v)$ and the closed neighborhood of $S$ is $N[S]=N(S) \cup S$. A set $S \subseteq V$ is a dominating set if $N[S]=V$, or equivalently, every vertex in $V \backslash S$ is adjacent to at least one vertex in $S$. An $i$-subset of $V(G)$ is a subset of $V(G)$ of cardinality $i$. Let $\mathscr{D}(G, i)$ be the family of dominating sets of $G$ which are $i$ subsets and let $d(G, i)=|\mathscr{D}(G, i)|$. The polynomial $D(G, x)=$ $\sum_{i=0}^{|V(G)|} d(G, i) x^{i}$ is defined as domination polynomial of $G$ [1]. This polynomial has been introduced by the author in his Ph.D. thesis in 2009 [2]. A root of $D(G, x)$ is called a domination root of $G$. More recently, domination polynomial has found application in network reliability [3]. For more information and motivation of domination polynomial and domination roots refer to $[1,2]$.

The join $G=G_{1}+G_{2}$ of two graphs $G_{1}$ and $G_{2}$ with disjoint vertex sets $V_{1}$ and $V_{2}$ and edge sets $E_{1}$ and $E_{2}$ is the graph union $G_{1} \cup G_{2}$ together with all the edges joining $V_{1}$ and $V_{2}$. The corona of two graphs $G_{1}$ and $G_{2}$, is the graph $G=$ $G_{1} \circ G_{2}$ formed from one copy of $G_{1}$ and $\left|V\left(G_{1}\right)\right|$ copies of $G_{2}$, where the $i$ th vertex of $G_{1}$ is adjacent to every vertex in the $i$ th copy of $G_{2}[4]$. the Cartesian product of two graphs $G$ and $H$ is denoted by $G \square H$, is the graph with vertex set $V(G) \cup V(H)$ and edges between two vertices $\left(u_{1}, v_{1}\right)$ and $\left(u_{2}, v_{2}\right)$ if and only if either $u_{1}=u_{2}$ and $v_{1} v_{2} \in E(H)$ or $u_{1} u_{2} \in E(G)$ and $v_{1}=v_{2}$.
In this paper, we study the domination polynomials of some graph operations.

\section{Main Results}

As is the case with other graph polynomials, such as chromatic polynomials and independence polynomials, it is natural to consider the domination polynomial of composition of two graphs. It is not hard to see that the formula for domination polynomial of join of two graphs is obtained as follows.

Theorem 1 (see [1]). Let $G_{1}$ and $G_{2}$ be graphs of orders $n_{1}$ and $n_{2}$, respectively. Then

$$
\begin{aligned}
D\left(G_{1}+G_{2}, x\right)= & \left((1+x)^{n_{1}}-1\right)\left((1+x)^{n_{2}}-1\right) \\
& +D\left(G_{1}, x\right)+D\left(G_{2}, x\right) .
\end{aligned}
$$

It is obvious that this operation of graphs is commutative. Using this product, one is able to construct a connected graph $G$ with the number of dominating sets $n$, where $n$ is an arbitrary odd natural number; see [5].

Let to consider the corona of two graphs. The following theorem gives us the domination polynomial of graphs of the form $H \circ K_{1}$ which is the first result for domination polynomial of specific corona of two graphs.

Theorem 2 (see [1]). Let $G$ be a graph. Then $D(G, x)=x^{n}(x+$ $2)^{n}$ if and only if $G=H \circ K_{1}$ for some graph $H$ of order $n$. 
It is easy to see that the corona operation of two graphs does not have the commutative property. The following theorem gives us the domination polynomial of $K_{1} \circ H$.

Theorem 3. For every graph $H$ of order $m, D\left(K_{1} \circ H, x\right)=$ $x(1+x)^{m}+D(H, x)$.

Proof. In each graph of the form $K_{1} \circ H$, we have two cases for a dominating set $S$.

Case 1. $S$ includes $u$ (the vertex originally in $K_{1}$ ) and an arbitrary subset of the $m$ vertices from the copy of $H$. The generating function for the number of dominating sets of graph in this case is $x(1+x)^{m}$.

Case 2. $S$ does not include $u$ and it is exactly a dominating set of $H$. In this case $D(H, x)$ is the generating function.

By addition principle, we have $D\left(K_{1} \circ H, x\right)=x(1+x)^{m}+$ $D(H, x)$.

The following theorem gives a formula for domination polynomial of corona products of two graphs.

Theorem 4. Let $G=(V, E)$ and $H=(W, F)$ be nonempty graphs of order $n$ and $m$, respectively. Then

$$
D(G \circ H, x)=\left(x(1+x)^{m}+D(H, x)\right)^{n} .
$$

Proof. By Theorem 3, it suffices to prove that $D(G \circ H, x)=$ $\left(D\left(K_{1} \circ H, x\right)\right)^{n}$. In the corona of two graphs $G$ and $H$, every vertex $u \in V$ of $G$ is adjacent to all vertices of the corresponding copy of $H$. So, we can delete all edges in $E$ in the corona. Therefore, the arising graph is the disjoint union of $|V|$ copies of the corona $K_{1} \circ H$. Therefore, $D(G \circ H, x)=$ $\left(D\left(K_{1} \circ H, x\right)\right)^{n}$.

As a consequence of the above theorem, we have the following corollary.

Corollary 5. (i) Let $G$ be a connected graph of order $n$. Then, $G=H \circ \bar{K}_{2}$, for some graph $H$, if and only if $D(G, x)=$ $x^{(n / 3)}\left(x^{2}+3 x+1\right)^{(n / 3)}($ see $[1])$.

(ii) Let $H$ be a graph of order $n$ and $G=H \circ \overline{K_{m}}$. Then $D(G, x)=\left(x(1+x)^{m}+x^{m}\right)^{n}$.

It is interesting that for the classification of graphs with exactly two, three, and four domination roots, we must consider some kinds of corona of two graphs. For more information, see [1].

To study more we need the following theorem.

Theorem 6 (see [2]). If $G$ has $t$ connected components $G_{1}$, $\ldots, G_{t}$, then $D(G, x)=\prod_{i=1}^{t} D\left(G_{i}, x\right)$.

Now we will consider the Cartesian product of two graphs. First we prove the following easy result.

Theorem 7. If $G$ has $t$ connected components $G_{1}, \ldots, G_{t}$, then

$$
D(G \square H, x)=\prod_{i=1}^{t} D\left(G_{i} \square H, x\right) .
$$

Proof. Since we have $\bigcup_{i=1}^{t} G_{i} \square H=\bigcup_{i=1}^{t}\left(G_{i} \square H\right)$, we have the result by Theorem 6 .

Despite the above property, it is difficult to determine the domination polynomial of this product, even in such simple cases as the grid graphs $P_{n} \square P_{m}$.

Now we consider another operation of two graphs. Let $G$ and $H$ be graphs, with $V(G)=\left\{v_{1}, \ldots, v_{n}\right\}$. The graph $G \diamond H$ formed by substituting a copy of $H$ for every vertex of $G$ is formally defined by taking a disjoint copy of $H, H_{v}$, for every vertex $v$ of $G$ and joining every vertex in $H_{u}$ to every vertex in $H_{v}$ if and only if $u$ is adjacent to $v$ in $G$.

The following result is also proven in [6, Lemma 3].

Theorem 8. For any graph $G, D\left(G \diamond K_{t}, x\right)=D\left(G,(1+x)^{t}-1\right)$.

Proof. Note that the closed neighborhood of the vertex $(u, v)$ of graph $G \diamond K_{t}$ is $\left(N_{G}[u], K_{t}\right)$. To make a dominating set of $G \diamond K_{t}$, suppose that $S \subset V(G)$ is a dominating set for $G$. It is easy to see that $\bigcup_{s \in S}\left\{(s, v) \mid v \in A_{s}\right\}$ is a dominating set of $G \diamond K_{t}$, where $\left\{A_{s} \mid s \in S\right\}$ is a family of arbitrary nonempty subsets of $V\left(K_{t}\right)$. Therefore, every $v \in S$ corresponds to all nonempty subsets of $G \diamond K_{t}$ which have the generating function $(1+x)^{t}-1$. So we have the result.

We would like to obtain some corollaries. We recall the following theorems.

Theorem 9 (see [7]). (i) For every $n \geq 4$,

$$
\begin{aligned}
D\left(P_{n}, x\right)=x[ & D\left(P_{n-1}, x\right) \\
& \left.+D\left(P_{n-2}, x\right)+D\left(P_{n-3}, x\right)\right]
\end{aligned}
$$

with the initial values $D\left(P_{1}, x\right)=x, D\left(P_{2}, x\right)=x^{2}+2 x, D\left(P_{3}\right.$, $x)=x^{3}+3 x^{2}+x$.

(ii) For every $n \geq 4$,

$$
\begin{aligned}
D\left(C_{n}, x\right)=x[ & D\left(C_{n-1}, x\right) \\
& \left.+D\left(C_{n-2}, x\right)+D\left(C_{n-3}, x\right)\right]
\end{aligned}
$$

with the initial values $D\left(C_{1}, x\right)=x, D\left(C_{2}, x\right)=x^{2}+2 x, D\left(C_{3}\right.$, $x)=x^{3}+3 x^{2}+3 x$.

Here we consider the graphs obtained by selecting one vertex in each of $n$ triangles and identifying them. Some call them Dutch Windmill graphs $[8,9]$ and friendship graphs.

Theorem 10. For every $n \in \mathbb{N}$,

$$
D\left(G_{3}^{n}, x\right)=\left(2 x+x^{2}\right)^{n}+x(1+x)^{2 n} .
$$

Proof. It is easy to see that $G_{3}^{n}$ is join of $K_{1}$ and $n K_{2}$. Now by Theorem 1, we have

$$
\begin{aligned}
D\left(G_{3}^{n}, x\right) & =D\left(K_{1}, x\right)+D\left(n K_{2}, x\right)+\left((1+x)^{2 n}-1\right) x \\
& =\left(2 x+x^{2}\right)^{n}+x(1+x)^{2 n} .
\end{aligned}
$$


Theorem 8 can be used to generalize recurrence relations for the domination polynomial of some families of graphs. For example, we state and prove the following theorem.

Theorem 11. (i) Suppose that $G_{n, t}=P_{n} \diamond K_{t}$. Then

$$
\begin{aligned}
D\left(G_{n, t}, x\right)= & \left((x+1)^{t}-1\right) \\
\times & {\left[D\left(G_{n-1, t}, x\right)+D\left(G_{n-2, t}, x\right)\right.} \\
& \left.+D\left(G_{n-3, t}, x\right)\right] .
\end{aligned}
$$

(ii) Suppose that $H_{n, t}=C_{n} \diamond K_{t}$. Then

$$
\begin{aligned}
D\left(H_{n, t}, x\right)= & \left((x+1)^{t}-1\right) \\
& \times\left[D\left(H_{n-1, t}, x\right)+D\left(H_{n-2, t}, x\right)\right. \\
& \left.+D\left(H_{n-3, t}, x\right)\right] .
\end{aligned}
$$

(iii)

$$
\begin{aligned}
D\left(G_{3}^{n} \diamond K_{t}, x\right)= & \left((1+x)^{2 t}-1\right)^{n} \\
& +\left((1+x)^{t}-1\right)(1+x)^{2 n t} .
\end{aligned}
$$

Proof. (i) From Theorem 8 , we have $D\left(G_{n, t}, x\right)=D\left(P_{n},(1+\right.$ $x)^{t}-1$ ). Now by Part (i) of Theorem 9 , we have the result.

(ii) From Theorem 8 , we have $D\left(H_{n, t}, x\right)=D\left(C_{n},(1+x)^{t}-\right.$ 1). Now by Part (ii) of Theorem 9 , we have the result.

(iii) From Theorem 8, we have $D\left(G_{3}^{n} \diamond K_{t}, x\right)=D\left(G_{3}^{n},(1+\right.$ $\left.x)^{t}-1\right)$. Now by Theorem 10 , we have the result.

\section{Conclusion}

In this paper, we studied the domination polynomials of some graph operations. There are some open problems which are interesting to consider.

(i) What is the basic formula for the domination polynomial of the Cartesian product of two graphs?

For two graphs $G$ and $H$, let $G[H]$ be the graph with vertex set $V(G) \times V(H)$ and such that vertex $(a, x)$ is adjacent to vertex $(b, y)$ if and only if $a$ is adjacent to $b$ (in $G$ ) or $a=b$ and $x$ is adjacent to $y$ (in $H$ ). The graph $G[H]$ is the lexicographic product (or composition) of $G$ and $H$ and can be thought of as the graph arising from $G$ and $H$ by substituting a copy of $H$ for every vertex of $G$. There is a main problem.

(ii) How can compute the domination polynomial of Lexicographic product of the two graphs?

\section{Acknowledgments}

The author would like to express his gratitude to the referees for his comments. The research was in part supported by a Grant from IPM (no. 91050015).

\section{References}

[1] S. Akbari, S. Alikhani, and Y.-h. Peng, "Characterization of graphs using domination polynomials," European Journal of Combinatorics, vol. 31, no. 7, pp. 1714-1724, 2010.
[2] S. Alikhani, Dominating sets and domination polynomials of graphs [Ph.D. thesis], Universiti Putra Malaysia, 2009.

[3] K. Dohmen and P. Tittmann, "Domination reliability," Electronic Journal of Combinatorics, vol. 19, no. 1, paper 15, 2012.

[4] R. Frucht and F. Harary, "On the corona of two graphs," Aequationes Mathematicae, vol. 4, pp. 322-325, 1970.

[5] S. Alikhani, "The domination polynomial of a graph at -1 ," Graphs and Combinatorics, 2012.

[6] J. Brown and J. Tufts, "On the roots of domination polynomials," Graphs and Combinatorics, 2013.

[7] S. Alikhani and Y.-H. Peng, "Dominating sets and domination polynomials of certain graphs. II," Opuscula Mathematica, vol. 30, no. 1, pp. 37-51, 2010.

[8] S. Alikhani and E. Deutsch, "Graphs with domination roots in the right half-plane," In press.

[9] http://mathworld.wolfram.com/DutchWindmillGraph.html. 


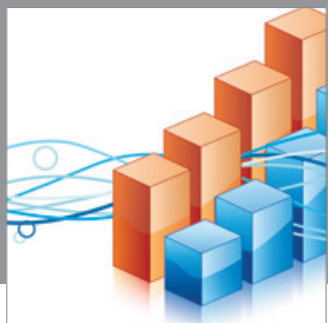

Advances in

Operations Research

mansans

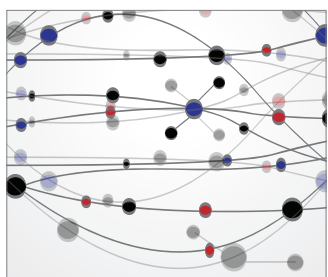

The Scientific World Journal
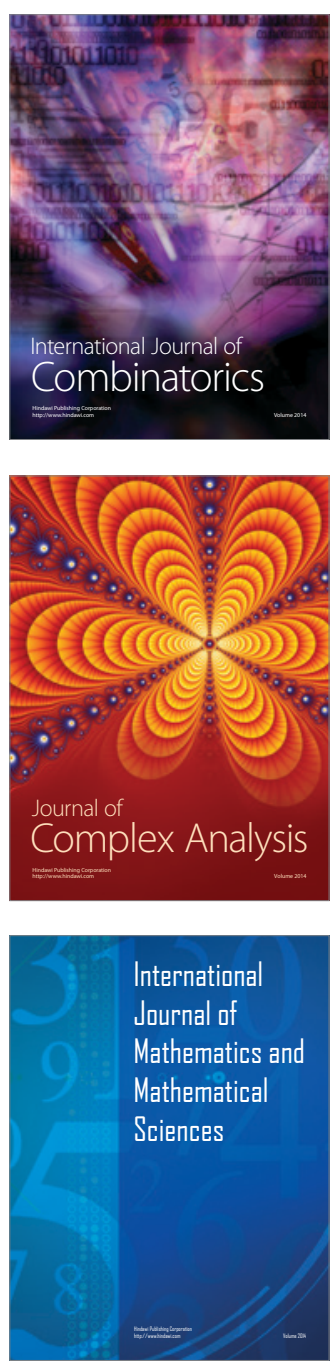
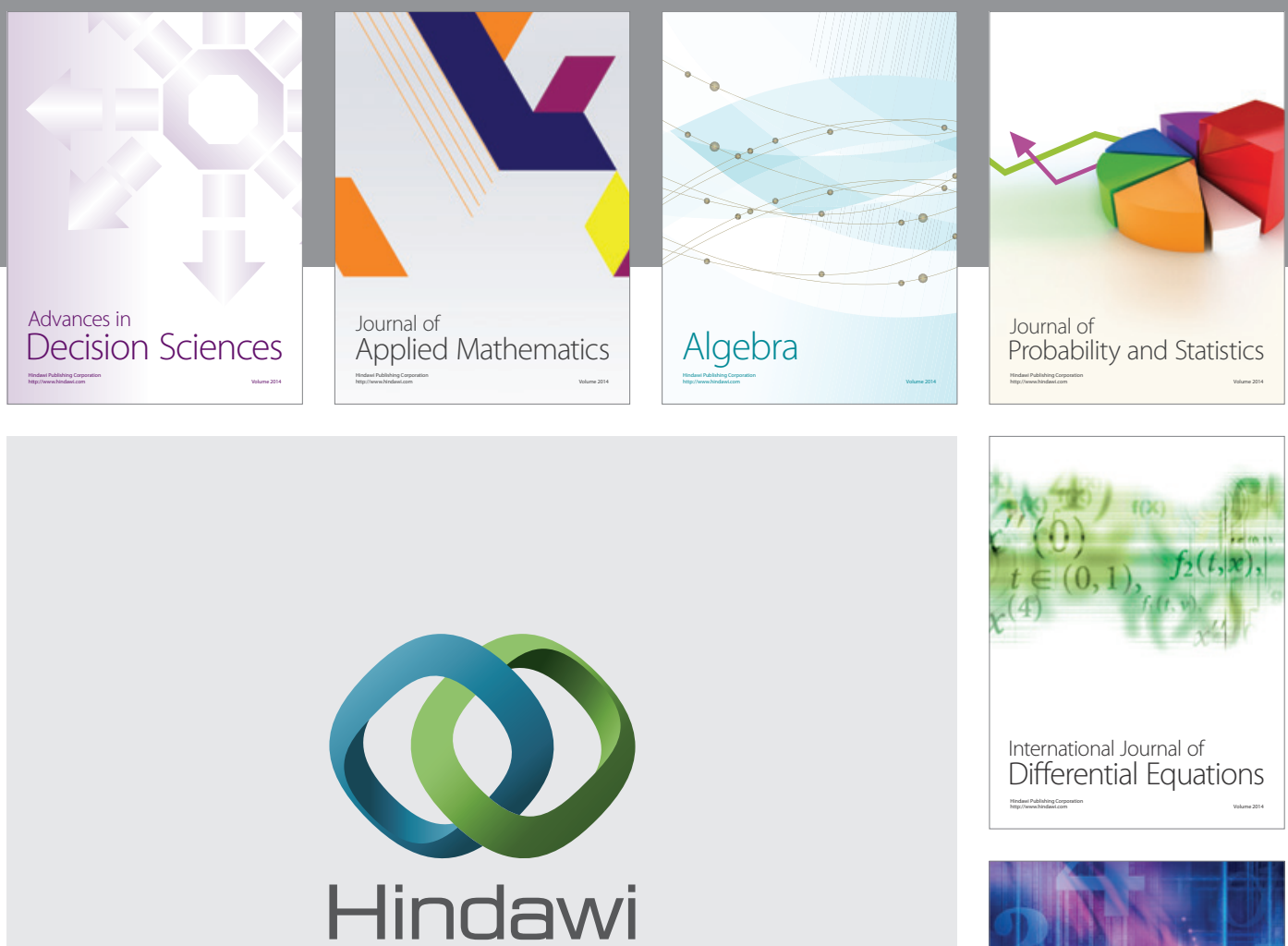

Submit your manuscripts at http://www.hindawi.com
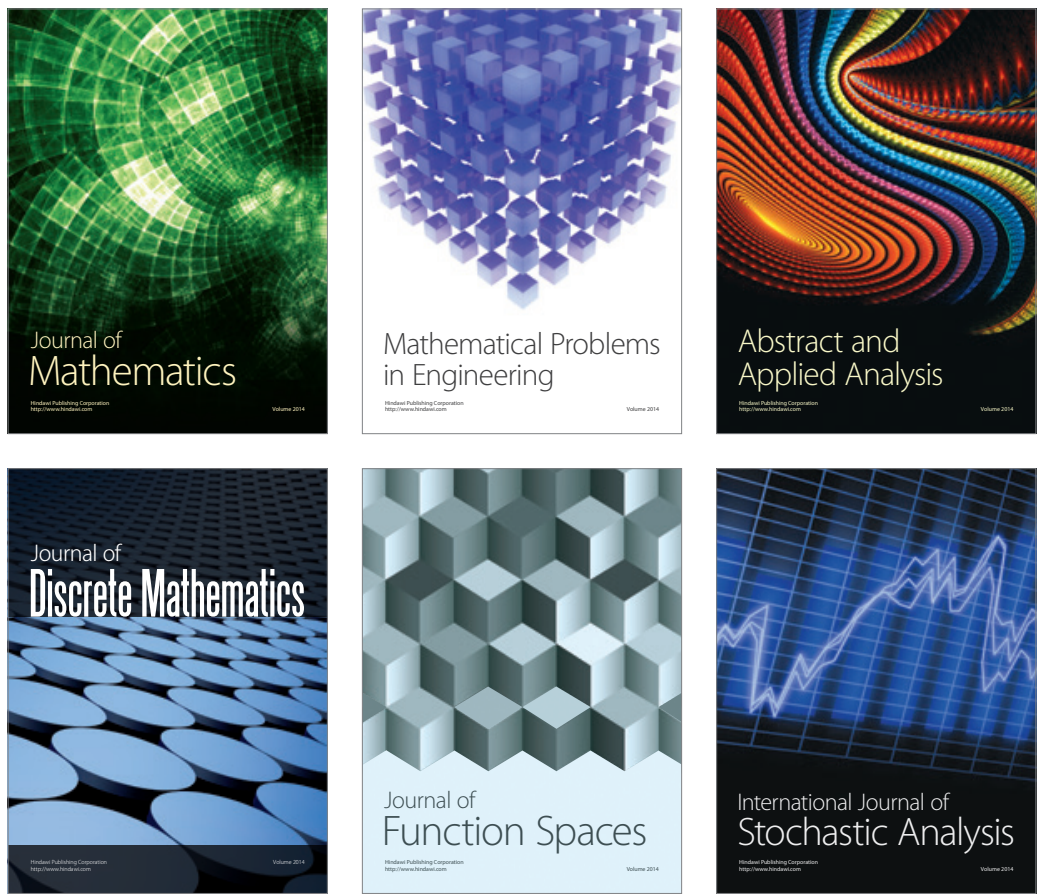

Journal of

Function Spaces

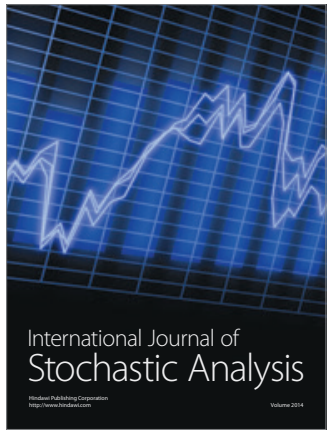

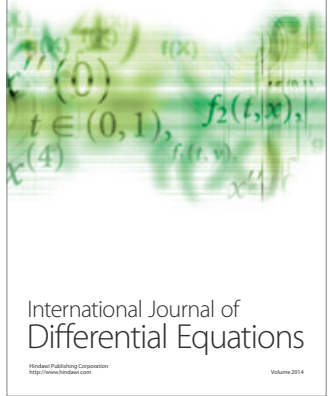
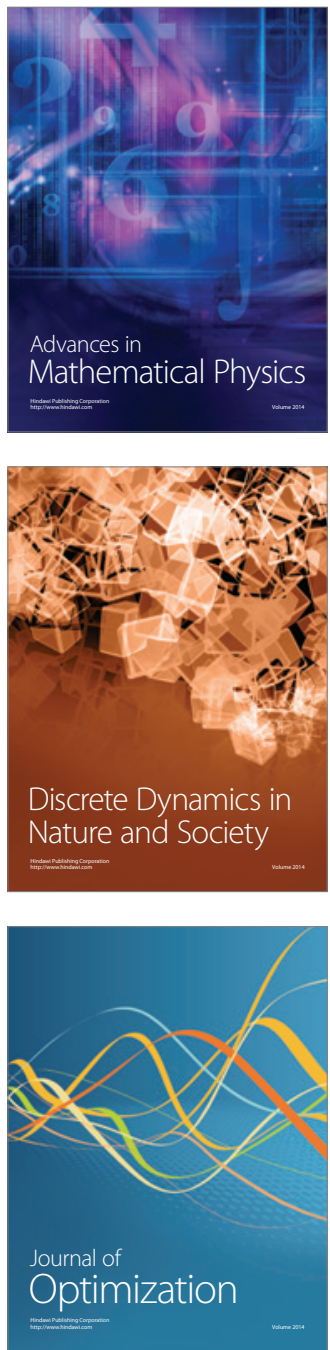